\title{
THE METRIZABLE LINEAR EXTENSIONS OF METRIZABLE SETS IN TOPOLOGICAL LINEAR SPACES
}

\section{DREWNOWSKI ${ }^{1}$}

ABSTRACT. Suppose a subset $X$ of a Hausdorff [locally convex] topological linear space $(E, \tau)$ is metrizable in its relative topology $\tau \mid X$. It is shown that if $\tau \mid X$ is separable, then there exists a metrizable [locally convex] linear topology $\tau_{0}$ on the subspace $V$ generated by $X$ such that $\tau_{0} \subset \tau \mid V$ and $\tau_{0}|X=\tau| X$ (Theorem 2). This is related to a recent result of Larman and Rogers which states that if, in addition, $X$ is locally bounded, then $\tau_{0}$ can be chosen to be normable (but then not necessarily $\tau_{0} \subset \tau \mid V$ ) (Theorem 1). It is then observed that $\tau_{0}|X=\tau| X$ does not mean the coincidence of the corresponding induced uniformities on $X$. However, this is the case if the invariant uniformity compatible with $\tau$ is metrizable on $X$ and $X$ is convex (Theorem 4).

Notation. $E=(E, \tau)$ denotes a (real or complex) Hausdorff topological linear space, $X$ a nonempty subset of $E, V$ the linear subspace of $E$ spanned by $X$.

$X$ is said to be locally bounded if, for each $x$ in $X$, there is a $\tau$-neighbourhood $C$ of the origin such that $(x+C) \cap X$ is bounded.

If $\lambda$ is a topology on a space containing a set $A$, then $\lambda \mid A$ denotes the topology on $A$ induced by $\lambda$.

Our purpose is to discuss the following theorem obtained recently by Larman and Rogers [4], and to simplify slightly its proof. Then we establish also a few related results.

Theorem 1. Suppose $E$ is locally convex, $X$ locally bounded and $\tau \mid X$ second countable (= metrizable and separable). Then it is possible to introduce a norm || on $V$ so that $\tau \mid X$ coincides with the relative topology of $X$ as a subset of the normed space $(V,||)$.

Received by the editors March 12, 1974 .

AMS (MOS) subject classifications (1970). Primary 46A05, 46B05; Secondary 54E 15, 54E35, 22A05.

Key words and phrases. Topological linear space, induced topology, second countable topology, metrizable linear topology, norm, induced uniformity, metrizable uniformity, convex set, absolutely convex hull.

1 This work was done while the author held a Postdoctoral Fellowship at the University of Florida, on leave from the A. Mickiewicz University, Poznan, Poland. 
Actually, in the original formulation of Theorem $1, X$ is assumed to contain 0 . That this condition is superfluous can be shown, apart from the proof of this theorem given below, as follows. Suppose Theorem 1 has been proved in the case $0 \in X$. Then, for an arbitrary $X$, choose any $x_{0}$ from $X$ and consider $X_{0}=X-x_{0}$. Let $V_{0}$ be the linear span of $X_{0}$. Since now $0 \in X_{0}$, there is a norm ||$_{0}$ on $V_{0}$ which induces $\tau \mid X_{0}$ on $X_{0}$. If $x_{0} \in V_{0}$, then $V=$ $V_{0}$ and we set ||$=||_{0}$. If $x_{0} \notin V_{0}$ then each $x \in V$ has a unique representation $x=v_{0}+t x_{0}, v_{0} \in V_{0}$, and we set $|x|=\left|v_{0}\right|_{0}+|t|$. In both cases the norm || coincides with ||$_{0}$ on $V_{0}$, and since translations are homeomorphisms, the topologies on $X=X_{0}+x_{0}$ induced by || and $\tau$ are identical.

As Professor C. A. Rogers explained in a letter dated November 20, 1973: "... A re-examination of the referee's example (see [4, p. 40]) shows that he did not actually prove the result we attributed to him. He actually disproved a stronger version of our Lemma 1 that we had originally used ..."."

We should note that the proof of Theorem 1 given in [4] is valid only if $E$ is real.

An analysis of the proof given by Larman and Rogers shows that the following three stages may be distinguished in it:

$1^{\circ}$. Construction of a metrizable locally convex topology $\tau_{1}$ on $V$ such that $\tau_{1} \subset \tau \mid V$.

$2^{\circ}$. Construction of semimetrizable locally convex topology $\tau_{2}$ on $V$ such that $\tau_{2} \subset \tau \mid V$ and $\tau_{2}|X=\tau| X$.

The topology $\tau_{0}=\tau_{1} \vee \tau_{2}$ on $V$ is metrizable and locally convex, $\tau_{0} C$ $\tau \mid V$ and $\tau_{0}|X=\tau| X$. Then the last step is

$3^{\circ}$. Construction of a norm required in Theorem 1 from a sequence of seminorms ||$_{n}$ defining $\tau_{0}$.

Moreover, it can be easily observed that in $1^{\circ}$ and $2^{\circ}$ the local boundedness of $X$ has not been used. This leads to

Theorem 2. Suppose $\tau \mid X$ is second countable. Then $(V, \tau \mid V)$ is a continuous image of a metrizable separable topological space, so that $r \mid V$ is fully Lindelöf. Hence there is a metrizable linear topology $\tau_{1}$ on $V$ such that $\tau_{1} \subset \tau \mid V$. Moreover, there exists a metrizable linear topology $\tau_{0}$ on $V$ such that $\tau_{0} \subset \tau \mid V$ and $\tau_{0}|X=\tau| X$. If, in addition, $\tau$ is locally convex, then also $\tau_{1}$ and $\tau_{0}$ can be chosen to be locally convex.

Proof. We can and do assume $E=V$. Let $\mathcal{C}$ be a base of open neighbourhoods of 0 in $(E, \tau)$, and $\mathcal{B}$ a countable base for $\tau \mid X$.

1 . To prove that $\tau \mid E$ is fully Lindelö, we proceed as in the proof of Theorem V.1.1 in [2]. Let $K$ denote the field of scalars of $E$, and $M$ the 
sum of the metrizable separable spaces $K^{n} \times X^{n}, n \in N$. Then $(E, \tau)$ is the image of $M$ under the continuous map $f$ whose restriction to $K^{n} \times X^{n}$ is defined by

$$
f\left(\left(t_{1}, \ldots, t_{n}\right),\left(x_{1}, \ldots, x_{n}\right)\right)=\sum_{i=1}^{n} t_{i} x_{i}
$$

Since $M$ is metrizable and separable, $(E, \tau)$ is fully Lindelöf. Since $E \backslash\{0\}=$ $\bigcup\{E \backslash \bar{C}: C \in \mathcal{C}\}$, there is a countable subfamily $\mathcal{C}_{1}$ of $\mathcal{C}$ such that $\cap \mathcal{C}_{1}=$ \{0\}. We can assume that $\mathcal{C}_{1}$ is a base at 0 for a linear topology, $\tau_{1}$, on $E$. Evidently, $\tau_{1}$ is as required in the theorem.

The existence of $r_{1}$ can be also proved in the folowing way (cf. [4]). If $A_{1}, \ldots, A_{n}$ are subsets of $E$, we define

$$
W\left(A_{1}, \ldots, A_{n}\right)=\bigcup_{j=1}^{n} \cup\left\{\sum_{i=1}^{n} t_{i} A_{i}:\left|t_{i}\right| \leq 1 \quad(i=1, \ldots, n),\left|t_{j}\right|=1\right\} .
$$

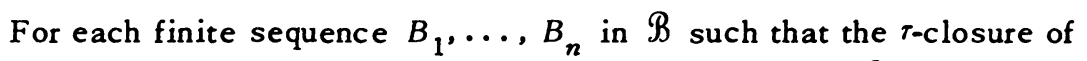
$W\left(B_{1}, \ldots, B_{n}\right)$ does not contain 0 , we choose $C$ in $\mathcal{C}$ so that $C \cap$ $W\left(B_{1}, \ldots, B_{n}\right)=\varnothing$. Let $\mathcal{C}^{\prime}$ be the countable subfamily of $\mathcal{C}$ obtained in this way. By adjoining to $\mathcal{C}^{\prime}$ some other members of $\mathcal{C}$ (if necessary), we readily define a countable subfamily $\mathcal{C}_{1}$ of $\mathcal{C}$ which will be a base at 0 for a linear topology, $\tau_{1}$, on $E$. Evidently $\tau_{1}$ is semimetrizable and $\tau_{1} \subset \tau_{\text {. We shall }}$ show in a moment that $\tau_{1}$ is Hausdorff, and so metrizable.

Take any $x \neq 0$ from $E$. Then we can find linearly independent elements $x_{1}, \ldots, x_{n}$ in $X$ such that

$$
x=\sum_{i=1}^{n} s_{i} x_{i}=s \sum_{i=1}^{n} t_{i} x_{i} ; \quad s=\sup \left|s_{i}\right|, \quad t_{i}=s_{i} / s .
$$

Since $x_{i}$ are linearly independent, there exists $C$ in $\mathcal{C}$ such that $C \cap$ $W\left(x_{1}+C, \ldots, x_{n}+C\right)=\varnothing$. For each $i$ the set $\left(x_{i}+C\right) \cap X$ is a neighbourhood of $x_{i}$ in $(X, \tau \mid X)$, hence there exists $B_{i}$ in $\mathscr{B}$ such that $x_{i} \in B_{i} \subset$ $\left(x_{i}+C\right) \cap X$. It follows that

$$
x / s \in W\left(B_{1}, \ldots, B_{n}\right) \subset W\left(x_{1}+C, \ldots, x_{n}+C\right)
$$

and $C \cap W\left(B_{1}, \ldots, B_{n}\right)=\varnothing$. Hence for some $C_{1}$ from $C^{\prime}$ we have $C_{1} \cap$ $W\left(B_{1}, \ldots, B_{n}\right)=\varnothing$, so that $(x / s) \notin C_{1}$. This proves that $\tau_{1}$ is Hausdorff.

$2^{\circ}$. We can suppose that each member of $B$ can be written as $(x+C+C)$ $\cap X$, where $x$ is taken from a countable subset of $X$ and $C$ from a countable 
subfamily $\mathcal{C}_{2}$ of $\mathcal{C}$. Clearly, we can also assume $\mathcal{C}_{2}$ to be a base at 0 for a linear topology, $\tau_{2}$, on $E$. It is then obvious that $\tau_{2}$ is semimetrizable, $\tau_{2} C$ $\tau$ and $\tau_{2}|X=\tau| X$.

Then the linear topology $\tau_{0}=\tau_{1} \vee \tau_{2}$ (for which the sets $C_{1} \cap C_{2}, C_{i}$ $\in \mathcal{C}_{i}(i=1,2)$, form a base at 0$)$ is as required in Theorem 2.

Proof of Theorem 1. In view of Theorem 2, there exists a sequence $\mid I_{n}$ of seminorms on $V$ which defines a metrizable locally convex topology $\tau_{0}$ on $V$ such that $\tau_{0} \subset \tau \mid V$ and $\tau_{0}|X=\tau| X$. Since $X$ is locally bounded and $\tau \mid X$ is Lindelöf, we can represent $X$ in the form $X=\bigcup_{n=1}^{\infty} X_{n}$, where each $X_{n}$ is open in $X$, bounded in $E$, and $X_{n} \subset X_{n+1}(n \in N)$. One can assume $|x|_{n} \leq 1$ for $x \in X_{n}(n \in N)$. [Otherwise replace ||$_{n}$ by $m_{n}^{-1}||_{n}, m_{n}=1+$ $\sup \left\{|x|_{n}: x \in X_{n}\right\}$.] Then the formula

$$
|x|=\sum_{n=1}^{\infty} 2^{-n}|x|_{n}
$$

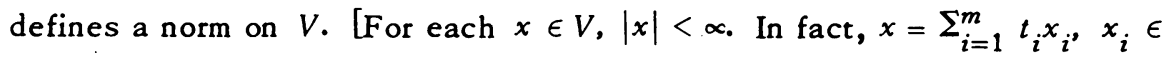
$X$, and for $k$ large enough all $x_{i}$ are in $X_{k}$. Then for $n \geq k$ we have $|x|_{n} \leq$ $\sum_{i=1}^{m}\left|t_{i}\right|=$ const, hence the series defining $|x|$ converges.]

Let $\nu$ denote the topology on $V$ determined by || . Evidently $\tau_{0} \subset \nu$.

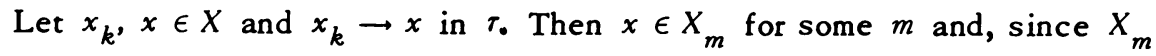
is open in $X$, we can assume that all $x_{k}$ are in $X_{m}$. It follows that

$$
\left|x-x_{k}\right| \leq \sum_{n=1}^{r}\left|x-x_{k}\right|_{n}+2^{-r+1}, \quad r \geq m, \quad k \geq 1 .
$$

Since $\left|x-x_{k}\right|_{n} \rightarrow 0(k \rightarrow \infty)$ for each $n$, it is now easy to conclude that $\left|x-x_{k}\right| \rightarrow 0$.

Thus $\nu|X \subset \tau| X=\tau_{0} \mid X$. Since $\tau_{0} \subset \nu$, also $\tau_{0}|X \subset \nu| X$.

A similar construction of a norm which induces a given topology on each me mber of a sequence of bounded sets can be found in [1].

An analogue of Theorem 2 for groups sounds as follows.

Theorem 2'. Suppose $(G, \gamma)$ is a Hausdorff topological abelian group, $A$ a subset of $G$, and $H$ the subgroup generated by $A$. If $\gamma \mid A$ is second countable, then $(H, \gamma \mid H)$ is a continuous image of a metrizable separable space, hence $(H, \gamma \mid H)$ is fully Lindelöf, and there exists a metrizable group topology $\gamma_{0}$ on $H$ such that $\gamma_{0} \subset \gamma \mid H$ and $\gamma_{0}|A=\gamma| A$.

The only major alteration that should be made in the proof of Theorem 2 is to use spaces $(-A)^{m} \times A^{n}$ in place of $K^{n} \times X^{n}$ [and sets of the form 


$$
W\left(t_{1}, \ldots, t_{n} ; A_{1}, \ldots, A_{n}\right)=\sum_{i=1}^{n} t_{i} A_{i}
$$

where each $t_{i}$ is either 1 or -1 , instead of $\left.W\left(A_{1}, \ldots, A_{n}\right)\right]$.

Also, it is seen that part $2^{\circ}$ of the proof of Theorem 2 can be easily modified to yield

Theorem 3. Let $\Gamma$ be a Hausdorff uniformity on a set $X$ and $\gamma$ the topology on $X$ associated with $\Gamma$. If $\gamma$ is second countable (=metrizable and separable), then there exists a metrizable uniformity $\Gamma_{0}$ on $X$ which is coarser than $\Gamma$ and compatible with $\gamma$.

In the setting of Theorem 2, $\gamma=\tau_{0}|X=\tau| X$, and as $\Gamma, \Gamma_{0}$ we consider naturally the uniformities induced on $X$ by the invariant uniformities compatible with $\tau$ and $\tau_{0}$, respectively. We are going to show that neither " $\Gamma \neq \Gamma_{0}$ " nor " $\Gamma$ is nonmetrizable" can be excluded.

To this aim, consider the example given in [4, pp. 46-48]: $E=l^{2}, \tau$ is the weak topology $\sigma\left(l^{2}, l^{2}\right)$, and $X=\left\{k e_{i}: k\right.$ is a nonzero integer, $\left.i \in N\right\}$, where $e_{i}$ denotes the $i$ th unit vector of $l^{2}$. Then $X$ is countable and $r \mid X$ is discrete, hence second countable, and as $\tau_{0}$ we can choose the topology of coordinate-wise convergence in $l^{2}$. Let $x_{n}=n^{2} e_{n}(n \in N)$. Then $x_{n} \rightarrow 0$ in $\tau_{0}$, hence the sequence $\left(x_{n}\right)$ is $\tau_{0}$-Cauchy. However, it is not $r$-Cauchy because for $v=(1 / n)_{n \in N}$ we have $\left\langle x_{2 n}-x_{n}, v\right\rangle=n, n \in N$. Hence the identity mapping $\left(X, \Gamma_{0}\right) \rightarrow(X, \Gamma)$ is not uniformly continuous, and so $\Gamma$ is not coarser than $\Gamma_{0}$.

Now suppose $\Gamma$ has a countable base. This is equivalent to the assumption that the point 0 of the set $Y=X-X$ has a countable base of neighbourhoods in the space $(Y, \tau \mid Y)$. Therefore there is a sequence $\left(v_{n}\right)$ in $l^{2}$ such that the sets

$$
U_{n}=\left\{y \in Y:\left|\left\langle y, v_{i}\right\rangle\right|<1 ; i=1, \ldots, n\right\}
$$

form a countable base at 0 in $(Y, \tau \mid Y)$. For each $n$, let $m_{n}$ be such that

$$
\left|\left\langle f_{n}, v_{i}\right\rangle\right|<1 / n \text { for } i=1, \ldots, n ; \quad f_{n}=e_{m_{n}} .
$$

Clearly we can assume $m_{1}<m_{2}<\ldots$. Let $v=\sum_{n=1}^{\infty} n^{-1} f_{n}$. Then for each $n$, $n f_{n} \notin U$, where $U=\{y \in Y:|\langle y, v\rangle|<1\}$. Hence none of $U_{n}$ is contained in the neighbourhood $U$ of 0 in $(Y, \tau \mid Y)$. It follows that $\Gamma$ is not metrizable. Since all $n f_{n}$ are in $X$, this argument shows also that $\tau \mid(X \cup\{0\})$ does not have a countable base at 0 , hence it is not metrizable. It follows that in the assertion of Theorem $2, X$ cannot be replaced by its 7 -closure. 
If $\alpha$ is a linear topology, we denote by $\Gamma(\alpha)$ the (unique) uniformity compatible with $\alpha$, and by $\Gamma(\alpha) \mid A$ the corresponding induced uniformity on a set $A$.

Theorem 4. Suppose $(E, \tau)$ is a Hausdorff locally convex space over reals and $X$ is a convex subset of $E$. Let $A$ denote the closed absolutely convex hull of $X$ and $W$ the linear subspace of $E$ generated by $A$. If $\Gamma(\tau) \mid X$ is metrizable, then there exists a metrizable locally convex topology $\mu$ on $W$ such that $\mu \subset \tau \mid W$ and $\Gamma(\mu)|A=\Gamma(\tau)| A$.

Proof. First observe that we can assume $0 \in X$. In fact, if $0 \notin X$, we can replace $X$ by $X-x_{0}$ and argue a little subtler than on $p .324$. Let $Y=X-X$. Since $\Gamma(\tau) \mid X$ is metrizable, there is a sequence $\left(U_{n}\right)_{n \in N}$ of absolutely convex open neighbourhoods of 0 in $(E, r)$ such that the sets $Y$ $\cap U_{n}$ form a base at 0 in $(Y, \tau \mid Y)$. Since we can assume that $U_{n+1}+U_{n+1} \subset$ $U_{n}(n \in N)$, there exists a locally convex topology $\nu$ on $E$ for which $\left(U_{n}\right)_{n \in N}$ is a base at 0 . Evidently $\nu$ is semimetrizable and $\nu \subset \tau$. Since $X \subset Y$ and $Y$ is absolutely convex, $A \subseteq \bar{Y}$. Also, since $\bar{Y} / 2 \subset A$, it is clear that $W$ is spanned by $\bar{Y}$. Now we shall show that the sets $\bar{Y} \cap U_{n}$ form a base at 0 in $(\bar{Y}, \tau \mid \bar{Y})$. Let $U$ be any closed neighbourhood of 0 in $(E, \tau)$. Then there is $U_{n}$ such that $Y \cap U_{n} \subset U$. Let $y \in \bar{Y} \cap U_{n}$. Then, given a neighbourhood $V$ of 0 in $(E, \tau),(y+V) \cap U_{n}$ is a $\tau$-neighbourhood of $y$, hence there is $x$ in $Y \cap U_{n}$ such that $x \in y+V$. It follows that $y \in\left(Y \cap U_{n}\right)+V \subset U+V$. Consequently, $y \in U$. Thus $\bar{Y} \cap U_{n} \subset U$.

Now it is quite obvious that the topology $\mu=\nu \mid W$ is Hausdorff, hence metrizable. By Grothendieck's lemma [3, 21.6(5)], both the identity mapping $(A, \Gamma(\tau) \mid A) \rightarrow(A, \Gamma(\mu) \mid A)$ and its inverse are uniformly continuous. Hence $\Gamma(\tau)|A=\Gamma(\mu)| A$. This completes the proof.

It is not clear to the author whether Theorem 4 is valid in complex spaces, as well as whether convexity of $X$ is necessary. The assumption that $X$ is convex can be omitted if $X$ is compact (see [5, Theorem 5.2], and [4, Theorem 2]), (added in proof) or precompact (see [6, Theorem 1.4]).

\section{REFERENCES}

1. A. Alexiewicz and $Z$. Semadeni, Linear functionals on two-norm spaces, Studia Math. 17 (1958), 121-140. MR 20 \#6644.

2. J. Hoffman-Jorgensen, The theory of analytic spaces, Various Publications Series, no. 10, Institute of Mathematics, Aarhus University, Denmark, 1970.

3. G. Köthe, Topologische lineare Räume. I, Die Grundlehren der math. Wissenschaften, Band 107, Springer-Verlag, Berlin, 1960; English transl., Die Grundlehren 
der math. Wissenschaften, Band 159, Springer-Verlag, New York, 1969. MR 24 \#A411; 40 \#1750.

4. D. G. Larman and C. A. Rogers, The normability of metrizable sets, Bull. London Math. Soc. 5 (1973), 39-48. MR 47 \#9217.

5. M. A. Rieffel, The Radon-Nikodym theorem for the Bochner integral, Trans. Amer. Math.Soc. 131 (1968), 466-487. MR 36 \#5297.

6. N. J. Kalton, Some forms of the closed graph theorem, Proc. Cambridge Philos. Soc. 70 (1971), 401-408. MR $46 \# 634$.

DEPARTMENT OF MATHEMATICS, UNIVERSITY OF FLORIDA, GAINESVILLE, FLORIDA 32611

Current address: Institute of Mathematics, A. Mickiewicz University, ul. Matejki 48/49, 60-769 Poznań, Poland 